\title{
Transient nanostrain detection in phi-OTDR using statistics-based signal processing
}

\author{
Hao Chen, Yang Xu, Sen Qian, Hai Yuan, and Lei Su
}

\begin{abstract}
Phase-sensitive optical time-domain reflectometry (Ф-OTDR) is capable of detecting acoustic emission induced small strain with high sensitivity. However, there is a tradeoff between sensitivity, bandwidth and detection range, which makes the detection of a transient weak signal challenging. In this work, we focus on transient weak signal detection using Ф-OTDR. To achieve this aim, we propose a cascaded statistics-based signal-processing framework in a $\Phi$-OTDR system to fetch the transient weak signal from a noisy background. Our framework is based on two key elements, including an estimator that is derived based on the probability characteristic of the Rayleigh backscattered light, and a time-frequency feature extraction process that maps the signal to the time-frequency domain. Using our statistics-based signal processing $\Phi-O T D R$, we demonstrate experimentally the detections of, firstly a weak persistent signal with a magnitude down to $4 \mathrm{n} \varepsilon$ and a frequency up to $40 \mathrm{kHz}$, and then a weak transient acoustic tone-burst signal. Our proposed scheme is promising for $\Phi$-OTDR performance improvement particularly in weak signal detections, and it will find new applications in the future systems.
\end{abstract}

Index Terms-Acoustic signal detection, Distributed detection, Rayleigh scattering, Strain measurement, Time domain reflectometry.

\section{INTRODUCTION}

A COUSTIC detection is important for nondestructive testing and structural diagnosis. There are a number of approaches to detect acoustic signals. Interferometry approaches are widely studied in acoustic detections, including Sagnac effect [1], Mach-Zehnder [2], Michelson [3, 4] and Fabry-Perot [5] interferometers. Fiber Bragg gratings are also used for acoustic detection, offering a quasi-distributed sensing capability with wavelength- or time-division multiplexing [6-9]. Notably, fully distributed fiber-optic strain sensors enable the measurement of acoustic signal along the entire-length of the fiber. Distributed dynamic strain sensors have been

This work was supported in part by the National Natural Science Foundation of China (Grant No. 51577149) and National Key Research and Development Program of China (Grant No. 2016YFB 0900704).

Hao Chen is currently pursuing a Ph.D. degree in electrical engineering in Xi'an Jiaotong University, Xi'an, China. (e-mail: chenhao49@ xjtu.edu.cn).

Yang $\mathrm{Xu}$ is with the School of Electrical Engineering, Xi'an Jiaotong University, Xi'an, China. (e-mail: xuyang@xjtu.edu.cn).

Sen Qian is with the Guangzhou Institute of Advanced Technology, Chinese Academy of Sciences, Guangzhou, China. (e-mail: sen.qian@giat.ac.cn).

Hai Yuan is with the Guangzhou Institute of Advanced Technology, Chinese Academy of Sciences, China. (e-mail: hai.yuan@ giat.ac.cn).

Lei Su is with the School of Engineering and Materials Science, Queen Mary University of London, London, UK. (e-mail: 1.su@qmul.ac.uk) demonstrated using Brillouin scattering via BOTDR [10], BOTDA [11] and BOCDA [12]. POTDR [13, 14] and Ф-OTDR [15] are also used to detect distributed vibrations and acoustic emissions. In particular, $\Phi$-OTDR technology relying on Rayleigh scattering is capable of detecting and locating the dynamic perturbations by monitoring the change of $\Phi$-OTDR traces. Although $\Phi$-OTDR has been studied extensively [16-20], the detection of a weak transient signal is still challenging [21]. Weak transient signal detection is important for various industrial applications, for example, electrical partial discharge (PD) is detrimental to power equipment diagnosis, the accompanying acoustic emissions can be generated with magnitude ranging from $\mathrm{mPa}$ to $\mathrm{hPa}$ and frequency spanning from tens of $\mathrm{Hz}$ to $\mathrm{MHz}$ [22]. Previous works in $\Phi$-OTDR [15, 23] utilize moving averaging algorithm to pre-processing the $\Phi$-OTDR traces, and then make a trace-by-trace comparison to locate the presence of any perturbation. This conventional procedure is suitable for the lower frequency vibration cases, and it requires a superimposed plotting of numerous curves, which is of less efficiency in the state-of-art $\Phi$-OTDR system.

In this research, we focus on the application of $\Phi$-OTDR to detect high-frequency transient weak signals. As introduced before, it is challenging to detect transient weak signal using $\Phi-O T D R$. To achieve this, we use coherent detection to collect the Rayleigh backscattered light and propose a signal processing framework to extract the weak signal from the background noises. The framework consists of a statistical estimator that is derived based on the probability model of the stochastic Rayleigh backscattered light and a time-frequency process that extracts the temporal and frequency features of the transient signal. The derivation of the estimator is achieved by applying Rician density function. The sensitivity is improved by the statistical estimator that models the small phase perturbations embedded in the dominating background noises in the $\Phi$-OTDR system. The paper is organized as follows. We first introduce the theory of our statistic-based signal processing methods, then carry out experiment investigations of the proposed approach in single-frequency perturbation and tone-burst signal detections, and finally evaluate our approach and compare it with other methods in discussions.

\section{II.THEORY AND SIGNAL PROCESSING}

\section{A. $\Phi$-OTDR signal characteristics}

$\Phi$-OTDR based distributed fiber sensing detects the interference pattern of Rayleigh backscattered (RBS) light 
generated from multiple randomly located scatters within a small section (can be regarded as a spatial resolution cell) of the fiber. As a coherent light pulse propagates through the fiber, RBS light is quasi-continuously generated by these scatters and part of the RBS light is reflected back (the scatters are assumed to be very small compared to the optical light wavelength).

The received light at the fiber input $z=0$ at an instant time $t$ is the summation of the interfering RBS light generated from a specific spatial resolution cell of the test fiber, expressed as $\left[v_{\mathrm{g}} t / 2-W, v_{\mathrm{g}} t / 2\right]$, where $v_{\mathrm{g}}$ is the group velocity of light in the fiber, $W=v_{g} \Delta t / 2$ is the OTDR spatial resolution, and $\Delta t$ is the probe pulse width. The RBS light field can be written in the form

$$
\left.E_{\mathrm{RBS}}(t)\right|_{z=0}=E_{0} e^{j \omega_{\mathrm{c}} t} e^{-2 \alpha \bar{z}} \sum_{z_{n}=v_{\mathrm{g}} t / 2-W}^{v_{\mathrm{g}} t / 2}\left[r_{n} e^{j \varphi_{n}} e^{j \theta_{\mathrm{p}}}\right]
$$

where $E_{0}$ is the input light field, $\omega_{\mathrm{c}}$ is the light frequency, $\alpha$ is the loss factor of the fiber, and $\bar{z}$ is the average position of the scatters $z_{n}$ inside the aforementioned spatial resolution cell. We assume here that the loss does not change considerably over the length of $W . r_{n}$ is the reflectance of the $n$-th scatter, and $\varphi_{n}=2 \beta z_{n}$ is the corresponding phase delay of the $n$-th scatter, $z_{n}$ is its position, and $\beta$ is the propagation constant of the light in the fiber. In addition, an extra phase $\theta_{\mathrm{p}}$ may be involved in the section of spatial resolution cell in which any external perturbation exists, and it only affects those scatters located within or after the perturbation position (denoted by $Z_{0}$ ).

$$
\theta_{\mathrm{p}}=\left\{\begin{array}{lc}
0 & v_{\mathrm{g}} t / 2-W \leq z_{n}<Z_{0} \\
2 \varphi_{\mathrm{p}} & Z_{0} \leq z_{n} \leq v_{\mathrm{g}} t / 2
\end{array}\right.
$$

The factor of 2 represents that the phase difference should be applied twice as the light passes the perturbation section twice during the forward and backward propagation.

Due to the rather weak RBS light in the optical fiber, coherent detection $[15,23]$ is used to increase the sensitivity and obtain a much better signal-to-noise ratio (SNR). The principle of coherent detection involves mixing the low-level RBS light field, as expressed in (1), with a higher-power local oscillator (LO) light field, $E_{\mathrm{LO}} \exp \left(j \omega_{\mathrm{LO}} t\right)$, at a $3 \mathrm{~dB}$ fiber coupler, and then using the balanced photodetector (BPD) to detect the interference components of the mixed signal. The output signal from the photodetector is proportional to the real part of the beating of the RBS light field with the LO laser light field, it can be calculated by

$$
\begin{aligned}
s(t) & =2 G R \Re\left\{E_{\mathrm{RBS}}(t) \cdot E_{\mathrm{LO}}^{*}(t)\right\} \\
& =2 G R E_{0} E_{\mathrm{LO}} A(t) \cos \left[\omega_{\mathrm{IF}} t+\varphi_{0}(t)+\theta_{\mathrm{p}}+\phi_{\mathrm{n}}\right]
\end{aligned}
$$

in which $G$ and $R$ are the photodetector-related voltage gain and responsivity factor, respectively, $\mathcal{R}$ denotes the real part operator, and $\omega_{\mathrm{IF}}=\omega_{\mathrm{c}}-\omega_{\mathrm{LO}}$ is the intermediate frequency shift between the RBS and LO light due to the introduction of an acousto-optic modulator (AOM). $A(t)$ and $\varphi_{0}(t)$ are the resultant amplitude and phase of a complex random phasor sum that is originated from the stochastic characteristic of RBS light in the fiber. According to the RBS light field function in (1), the resultant amplitude and phase of the RBS light field can be expressed as follows:

$$
A(t) e^{j \varphi_{0}(t)}=\sum_{z_{\mathrm{n}}} r_{\mathrm{n}} e^{j \varphi_{\mathrm{n}}}
$$

Note that each of the phasor components $r_{n}$ and $\varphi_{n}=2 \beta z_{n}$ in the phasor summation are related to the positions of scatters in a particular spatial resolution cell $\left[v_{\mathrm{g}} t / 2-W, v_{\mathrm{g}} t / 2\right]$, thus both the resultant amplitude $A(t)$ and phase $\varphi_{0}(t)$ are the functions of the receiving time $t$. The last two phase terms in (3), $\theta_{\mathrm{p}}$ and $\phi_{\mathrm{n}}$, represent the acoustic perturbation induced phase shift and phase noise of laser and $\Phi$-OTDR system respectively, which will be accounted for later.

We notice that the constant coefficients and the time dependence terms in the received signal in (3) are fixed values, except that the resultant amplitude and phase in the summation notation of (4) will follow a statistical probability function. According to the random phasor sums theory [24], when we calculate the total complex amplitude of the light wave that arises as a result of scattering by a collection of small, independent scatters, as shown in (4), the real and imaginary parts of the resultant phasor both are asymptotically Gaussian random variables for a very large numbers of scatterers, with zero mean and equal variance $\sigma^{2}$. Then, the amplitude or instantaneous envelope of the received signal obeys Rayleigh statistics, with a probability density function (PDF) of

$$
p_{s A}(A)=\frac{A}{\sigma^{2}} \exp \left(-\frac{A^{2}}{2 \sigma^{2}}\right)
$$

for $A>0$, and $p_{S A}(A)$ is zero otherwise. A preliminary test was conducted to verify the statistical characteristic of the $\Phi$-OTDR received signal in absence of any exerted perturbation onto the fiber. The test result shown in Fig. 1(a) indicated the amplitude of the received signal $s(t)$ is a Rayleigh variate, as evidenced by the amplitude histogram according to the Rayleigh PDF fitting curve.

We also note that the received signal is proportional to the amplitude of light field, rather than the light power as used in the direct-detection approach. Fig. 1(b) demonstrates the linear relationship between the received signal and the LO field amplitude, not the power. In our experiment, a series of optical attenuators (with attenuation ranging from $0 \mathrm{~dB}$ to $-25 \mathrm{~dB}$ ) is inserted into the LO light path in the coherent detection setup, to vary the LO field amplitude. The optical amplitude of the LO light is typically much higher than that of the RBS light, and can therefore serve as a gain factor for the received signal. With a sufficiently strong LO field, the SNR of coherent detection can reach the shot-noise limit even for weak signal detection.

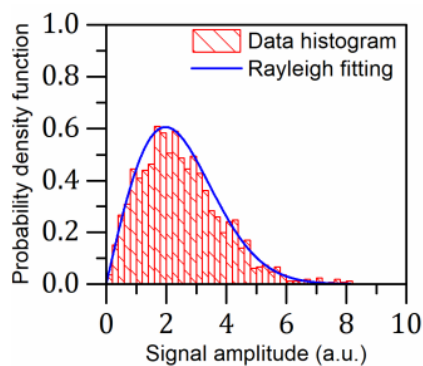

(a) Histogram of the $\Phi$-OTDR received signal and fitted Rayleigh distribution

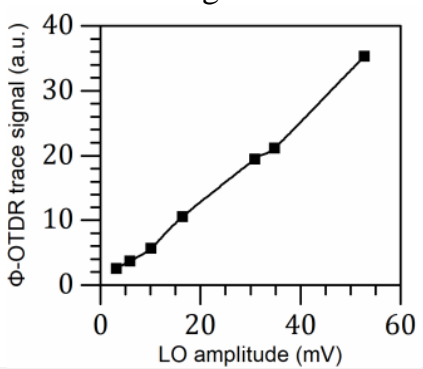

(b) The mean $\Phi$-OTDR received signal exhibits a linear dependence on the amplitude of the LO light
Fig. 1. Characteristics of the $\Phi-O T D R$ received signal with coherent detection. 


\section{B. Signal processing framework I: a statistics estimator}

The high sensitivity of $\Phi-O T D R$ is due to the mutual interference effect of the RBS light from various scatters, as presented in the random phasor sums in (4). The signal amplitude in proportion to $A(t)$ is determined by the phase relations of $\varphi_{\mathrm{n}}$ among the various random scatters. Therefore, the phase stability and coherence of the laser is essential to $\Phi-O T D R$ techniques, and the impact of laser phase noise on the phase modulation in the interference signal should be considered. We then write (3) in a more concise form:

$$
s(t)=A \cos \left[\left(\omega_{\mathrm{IF}} t+\varphi_{0}\right)+\theta_{\mathrm{p}}+\phi_{\mathrm{n}}\right]
$$

Here, we ignore the constant factors in (3) for simplicity, and $\varphi_{0}$ is the initial phase related to the original positions of the numerous scatters, which is a random variable uniformly distributed in the range $(-\pi, \pi)$.

Rewriting (6) by trigonometric operations, we obtain:

$$
\begin{aligned}
& s(t)=s^{0}(t)-\Delta s(t) \\
& s^{0}(t)=A \cos \left(\omega_{\mathrm{IF}} t+\varphi_{0}\right) \\
& \Delta s(t)=2 A \sin \frac{\theta_{\mathrm{p}}+\phi_{\mathrm{n}}}{2} \sin \left(\omega_{\mathrm{IF}} t+\varphi_{0}+\frac{\theta_{\mathrm{p}}+\phi_{\mathrm{n}}}{2}\right)
\end{aligned}
$$

We notice that the instantaneous value of the received signal $s(t)$ may have an increment or decrement component $\Delta s(t)$, which is a simultaneously amplitude- and phase-modulated signal related to the exerted phase perturbation $\theta_{\mathrm{p}}$ to be determined plus the phase noise term $\phi_{\mathrm{n}}$. This increment signal can be used to retrieve the phase perturbation from the fluctuating and noisy reference signal in the statistical manner as described below.

From (7), the modulated function in amplitude varies much more slowly with $t$ than the $\omega_{\mathrm{IF}}$ spectrum component, which can be derived from the quadratic sum of the increment signal $\Delta s(t)$ and its conjugate function $\Delta \hat{s}(t)$ by Hilbert transform:

$$
\begin{aligned}
& \Delta \hat{s}(t)=2 A \sin \frac{\theta_{\mathrm{p}}+\phi_{\mathrm{n}}}{2} \cos \left(\omega_{\mathrm{IF}} t+\varphi_{0}+\frac{\theta_{\mathrm{p}}+\phi_{\mathrm{n}}}{2}\right) \\
& |\Delta s(t)|=2 A \sin \frac{\theta_{\mathrm{p}}+\phi_{\mathrm{n}}}{2}=\left[\Delta s^{2}(t)+\Delta \hat{s}^{2}(t)\right]^{1 / 2}
\end{aligned}
$$

Under the small phase shift condition, the envelop of the amplitude-modulated function in (8), follows the approximation below:

$$
|\Delta s(t)| \approx A\left(\theta_{\mathrm{p}}+\phi_{\mathrm{n}}\right)
$$

This approximation holds for $\left[\theta_{\mathrm{p}}+\phi_{\mathrm{n}}\right] / 2 \leq 0.08 \pi$ within $1 \%$ errors.

Suppose we are detecting the particularly weak dynamic perturbation, such as the acoustic emission wave caused by the electrical partial discharge, and then, the increment signal envelop takes the form:

$$
|\Delta s(t)|=A \cdot\left[2 P \cos \left(2 \pi f_{\mathrm{a}} t\right)+\phi_{\mathrm{n}}\right]
$$

where $P$ is the amplitude of the phase shift function $\theta_{\mathrm{p}}(t)$ caused by an acoustic emission wave at a single frequency $f_{\mathrm{a}}$, and the factor of 2 denotes the round-trip effect of phase shift as shown in (2). Note that the time $t$ here is supposed in a long time scale compared to $1 / \Delta v_{\mathrm{a}}$, in which $\Delta v_{\mathrm{a}}$ is the acoustic perturbation bandwidth.

In (10), the Rayleigh distributed variate $A$ can be considered essentially constant over the time interval $\left(2 \pi / \omega_{\mathrm{IF}}\right)$ for a short section of the fiber under acoustic perturbation. In $\Phi-O T D R$ detection, without any phase perturbation the received signal-trace function $s(t)$ remains the same expression. However, the phase noise in the fiber sensor system is nonnegligible in different measurements. These factors include both the random frequency fluctuations of the narrow linewidth laser and the physical noise that arises from operation stability of laser sources and fiber-optic links due to thermal dynamical and mechanical fluctuations. Therefore, we hypothesize that the minimum detectable phase sensitivity is determined the laser frequency noise in $\Phi$-OTDR system. For a narrow linewidth laser, the laser phase undergoes random fluctuations with time, the phase fluctuations is a zero-mean Gaussian distribution with a variance of [25]

$$
\sigma_{\mathrm{n}}^{2}=E\left\{\phi_{\mathrm{n}}(t) \phi_{\mathrm{n}}(t-\Delta t)\right\}=2 \pi \Delta f_{\mathrm{L}} \Delta t
$$

where $\Delta f_{\mathrm{L}}$ is the full width at half maximum (FWHM) linewidth of the semiconductor laser and $\Delta t$ is the pulse width of the light probing the $\Phi-O T D R$ system.

In terms of statistics, when the laser phase noise is white Gaussian noise with variance of $\sigma_{\mathrm{n}}^{2}$, it can be written in the Fourier series representation as

$$
\phi_{\mathrm{n}}(t)=\sum_{n=1}^{N} c_{n} \cos \left(2 \pi f_{n} t-\psi_{n}\right)
$$

where $f_{n}$ is the $n$-th frequency component of the phase noise because it is white-noise, $c_{n}=\sqrt{2} \sigma_{\mathrm{n}} / \sqrt{N}, \psi_{1}, \cdots \psi_{n}$ are independent random phase angles uniformly distributed over the phase range $[0,2 \pi]$. In this representation the Gaussian-like phase noise is regarded as the sum of a large number of sinusoidal components with fixed amplitudes but random phases in the frequency domain.

Now we consider the distribution of the increment signal envelop function in (10). As stated before, the Rayleigh variate $A$ can be treated as constant on a short section of the fiber, by supposing that the frequency band associated with $\phi_{\mathrm{n}}$ is relatively narrow and contains the acoustic wave frequency $f_{\mathrm{a}}$. This is reasonable for the case of acoustic emission detection which we are interested in. The phase noise with the representation in (12) can be resolved into two components, one is "in phase" and the other is "in quadrature" with the sine wave $P \cdot \cos \left(2 \pi f_{\mathrm{a}} t\right)$,

$$
\begin{aligned}
\phi_{\mathrm{n}}(t) & =\sum_{n=1}^{N} c_{n} \cos \left[2 \pi\left(f_{n}-f_{\mathrm{a}}\right) t-\psi_{n}+2 \pi f_{\mathrm{a}} t\right] \\
& =I_{\mathrm{c}} \cos \left(2 \pi f_{\mathrm{a}} t\right)-I_{\mathrm{s}} \sin \left(2 \pi f_{\mathrm{a}} t\right)
\end{aligned}
$$

where

$$
\begin{aligned}
& I_{\mathrm{c}}=\sum_{n=1}^{N} c_{n} \cos \left[2 \pi\left(f_{n}-f_{\mathrm{a}}\right) t-\psi_{n}\right] \\
& I_{\mathrm{s}}=\sum_{n=1}^{N} c_{n} \sin \left[2 \pi\left(f_{n}-f_{\mathrm{a}}\right) t-\psi_{n}\right]
\end{aligned}
$$

Thus, the increment signal envelop $|\Delta s(t)|$ can be written as 


$$
\begin{aligned}
|\Delta s(t)| & =A\left[\left(2 P+I_{\mathrm{c}}\right) \cos \left(2 \pi f_{\mathrm{a}} t\right)-I_{\mathrm{s}} \sin \left(2 \pi f_{\mathrm{a}} t\right)\right] \\
& =A \cdot R \cos \left(2 \pi f_{\mathrm{a}} t+\delta\right)
\end{aligned}
$$

with the signal amplitude $R$ given by

$$
R=\sqrt{\left(2 P+I_{\mathrm{c}}\right)^{2}+I_{\mathrm{s}}^{2}}
$$

where $I_{\mathrm{c}}$ and $I_{\mathrm{s}}$ are distributed normally and centered at zero with a variance equal to $\overline{I_{\mathrm{c}}^{2}}=\overline{I_{\mathrm{s}}^{2}}=\sigma_{\mathrm{n}}^{2}$. Therefore, $R$ is also a random variable that follows a Rician distribution for small signal of the amplitude $P$. The probability density function of Rican distribution is [24],

$$
P_{|\Delta s|}(R)=\frac{R}{\sigma_{\mathrm{n}}^{2}} \exp \left[-\frac{R^{2}+4 P^{2}}{2 \sigma_{\mathrm{n}}^{2}}\right] I_{0}\left(\frac{2 R P}{\sigma_{\mathrm{n}}^{2}}\right)
$$

where $I_{0}$ is the Bessel function of order zero with imaginary argument. The mean value of $R$ is calculated as

$$
\bar{R}=\sqrt{\frac{\pi}{2}} \sigma_{n} \exp \left(-\frac{r}{2}\right)\left[(1+r) I_{0}\left(\frac{r}{2}\right)+r I_{1}\left(\frac{r}{2}\right)\right]
$$

in which $r=2 P^{2} / \sigma_{\mathrm{n}}^{2}$ denotes the ratio of signal power to noise power according to (10), $I_{0}(r)$ and $I_{1}(r)$ denote the zeroth- and first-order scaled modified Bessel functions of the first kind, respectively.

The statistical estimator $\bar{R}$ can be used to evaluate the presence of weak acoustic perturbation in the $\Phi$-OTDR system. Curves showing the relationship between the statistical estimator $\bar{R}$ and the phase amplitude $P$ due to acoustic wave perturbation under different laser phase noise with variance of $\sigma_{\mathrm{n}}^{2}$ is shown in Fig. 2. The laser phase noise level sets the minimum detectable acoustic phase shift, as depicted by the circled dots in each of the calculated curves. The circled dots are located at the acoustic phase shift abscissas, at which the ratio of the acoustic signal power to the laser noise power $r=2 P^{2} / \sigma_{\mathrm{n}}^{2}$ equals to 1 . For instance, the minimum phase sensitivity can be up to $0.018 \pi$ for the curve (green line) with a phase noise level of $\sigma_{\mathrm{n}}^{2}=0.002 \pi$, and the associated statistical estimator value, calculated by setting $r=1$ in (18), is $\bar{R} \approx 1.813 \sigma_{\mathrm{n}}$. When there is no acoustic perturbation, the statistical estimator reduces to a simple expression $\sqrt{\pi / 2} \sigma_{\mathrm{n}}$. Thus, at the minimum acoustic phase sensitivity, the statistical estimator $\bar{R}$ can achieve a SNR of $3.2 \mathrm{~dB}$. The statistical estimator may exhibit asymptotic saturation as the phase shift approaches toward $\pi / 2$ because of the sinusoidal function in the signal envelope.

Because the estimator treats the time series of $\Phi$-OTDR traces as statistical variables, the information of the signal dynamics is no longer preserved. However, the signal signatures can be recovered by a cascaded time-frequency estimator, which will be discussed in the following sections.

\section{Signal processing framework II: time-frequency estimator}

In $\Phi$-OTDR techniques, the perturbation acoustic waveform can be reconstructed in the time domain with the light pulses repetitively probing the test fiber. However, due to Rayleigh fading effect and the laser noise in $\Phi$-OTDR system, the reconstructed waveform may exhibit intense fluctuations in the time domain. It has been recognized that the use of time-

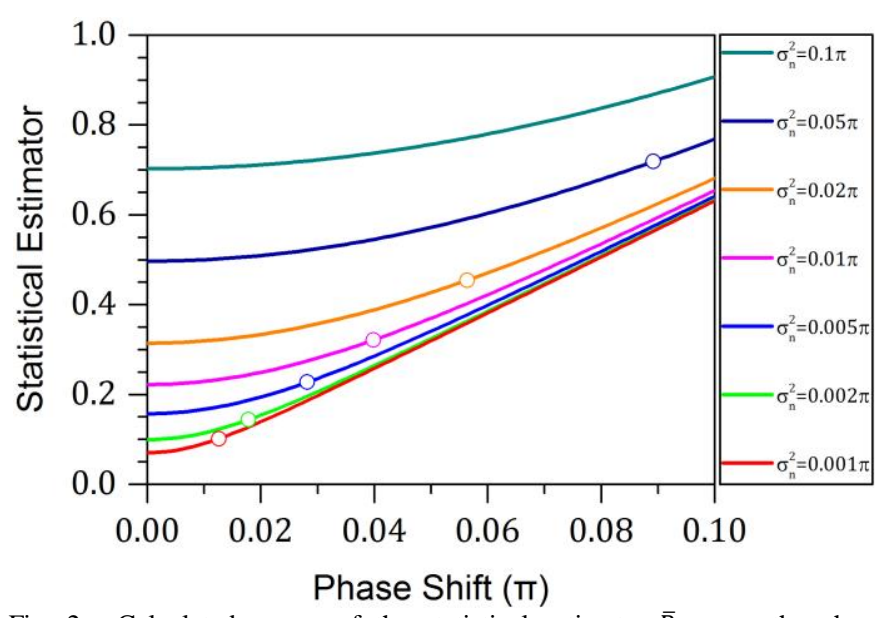

Fig. 2. Calculated curves of the statistical estimator $\bar{R}$ versus the phase amplitude $P$ caused by a single-tone acoustic waveform under different laser phase noise with variance of $\sigma_{\mathrm{n}}^{2}$. Supposing the Rayleigh variate $A=1$.

domain or frequency-domain information alone is often inadequate for characterizing the acoustic transient features, especially for a weak and transient signal submerged in noises. In search of a time-frequency analysis method, we find that Gabor spectrogram, developed in [26], to be the most suitable for providing the temporal and frequency features of the desired transient signal. For the signal of interest, $p(t)$, the Gabor representation of $p(t)$ using the window function $g(t)$ is

$$
p(t)=\sum_{m, n=-\infty}^{\infty} C_{m n} \cdot \mathrm{g}_{m n}(t)
$$

where

$$
\mathrm{g}_{m n}(t)=\mathrm{g}(t-m T) e^{j n \Omega t}
$$

is the time-shifted window function at the single frequency, $T$ and $\Omega$ represent the time and frequency sampling intervals, respectively, and $C_{m n}$ is the Gabor coefficient, which can be regarded as the measure of the similarity between the underlying signal $p(t)$ and each of the individual Gabor elementary bases $g_{m n}(t)$. For the detection of an acoustic transient signal, the Gabor window function $\mathrm{g}(t)$ can be chosen as the one-sided exponential function,

$$
\mathrm{g}(t)=\sqrt{2 \alpha} \exp (-\alpha t) u(t)
$$

where $u(t)$ is the unit step function and $\alpha$ is the damping factor.

After decomposing the reconstructed acoustic signal as a linear combination of the Gabor elementary functions, because of the quadrature of them, the Gabor coefficient matrix $C_{m n}$ is determined. Each elementary function locates a different time and frequency point $(m T, n \Omega)$ in the time-frequency domain, thus the Gabor representation of the signal involves the time-varying spectral contents that may be latent in the original transient waveform. Based on the instantaneous frequency concept, a time-frequency estimator is valid for tracking the transient signal signatures, as defined in

$$
f_{\mathrm{p}}(m T)=\frac{\sum_{n} n \Omega \cdot\left|C_{m n}\right|^{2}}{\sum_{n}\left|C_{m n}\right|^{2}}
$$

This time-frequency estimator is beneficial in characterizing the start and end of acoustic bursts, and it also has a better SNR 
by suppressing the white noise spectrum that spans beyond the acoustic signal bands.

\section{EXPERIMENT AND RESULTS}

\section{A. Nanostrain test with $\Phi-O T D R$}

The experimental configuration of $\Phi$-OTDR based on coherent detection scheme was shown in Fig. 3. An external cavity semiconductor laser with a wavelength of $1550 \mathrm{~nm}$ and narrow linewidth of $50 \mathrm{kHz}$ was used as a highly coherent light source, a key component in the $\Phi$-OTDR sensing system. The laser light was split by a $2 \times 2$ 90:10 fiber coupler (FC1) into two parts. One light path (with a coupling ratio of $10 \%$ ) acted as a reference called the LO light in coherent detection, and the other light path (with a coupling ratio of 90\%) was injected into an AOM, in which a sequence of repetitive light pulses was generated and the pulsed light gained an extra frequency shift $(\Delta v=200 \mathrm{MHz})$. After passing through an erbium-doped fiber amplifier (EDFA) for insertion-loss compensation and pulsed-light amplification, the pulse sequence was sent into the fiber under test via a circulator. The RBS light was generated at random positions along the whole length of the fiber where dielectric discontinuities served as various scatters. The RBS light propagated backwards to the input port of the fiber and was routed by the circulator again, and then the RBS light was mixed with the LO light at the second fiber coupler (FC2, 50:50), interfering with each other. Finally, through a BPD that removed the DC component and amplified the intermediate frequency component at $200 \mathrm{MHz}$, the interference light power was transformed into an electric signal and acquired by a highspeed data acquisition (DAQ) digitizer.

In the experiment, the AOM driven by an arbitrary waveform generator $(\mathrm{AWG})$ produced repetitive light pulses with a pulse repetition period of $10 \mu$ s and a pulse width of 31.2 ns. This meant that the system was capable of interrogating a 1000-m fiber with a spatial resolution of $3.12 \mathrm{~m}$. The fiber under test in the experiment was $764 \mathrm{~m}$ in length, consisting of three sections with lengths of $512 \mathrm{~m}, 4 \mathrm{~m}$ and $248 \mathrm{~m}$ respectively. The $4 \mathrm{~m}$ section of the fiber was wrapped around a piezoelectric actuator and served as the sensing section, whereas the other sections of fiber were placed inside an adiabatic and acoustic isolated chamber to suppress the environmental acoustic noises.

In order to precisely control the strain exerted on the fiber, a piezoelectric actuator customized by Fuji Ceramics was used in association with a programmable voltage source. The outer diameter of the piezoelectric ring is $100 \mathrm{~mm}$, and a section of bend-insensitive single-mode fiber was wrapped around the ring device with 10 turns. An off-the-shelf Mach-Zehnder interferometer was utilized to calibrate the dynamic strains induced on the fiber under different driven frequencies in the acoustic bands spanning from $10 \mathrm{kHz}$ to $200 \mathrm{kHz}$. The measurement result showed that below the resonance frequency of $160 \mathrm{kHz}$, the piezoelectric actuator generated dynamic strains with a flat spectral response. Specifically, by driving the actuator with a $1 \mathrm{Vpp}$ sinusoidal voltage, dynamic strain of approximately $1 \mathrm{n} \varepsilon$ in amplitude was generated on the fiber, corresponding to a phase shift of $4.6 \times 10^{-3} \pi$, which proportionally decreased to a $0.1 \mathrm{n} \varepsilon$ strain and a $4.6 \times 10^{-4} \pi$ phase shift for a $0.1 \mathrm{Vpp}$ drive voltage.

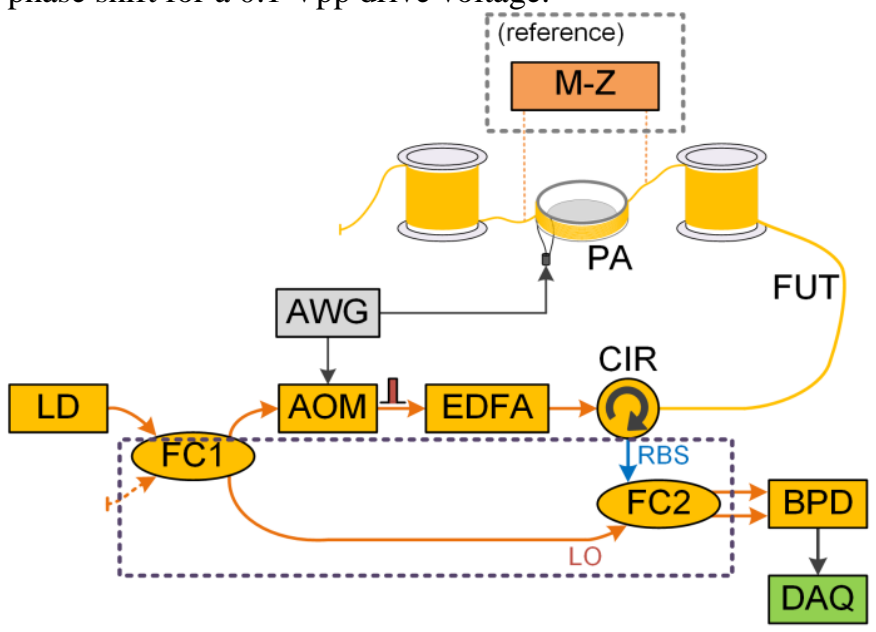

Fig. 3. Schematic configuration of $\Phi$-OTDR based on coherent detection. Acronyms: LD - laser diode; FC1 and FC2 - fiber coupler; AOM acousto-optic modulator; EDFA - erbium-doped fiber amplifier; CIR - fiber circulator; BPD - balanced photodetector; AWG - arbitrary waveform generator; DAQ - data acquisition; FUT - fiber under test; PA - piezoelectric actuator; $\mathrm{M}-\mathrm{Z}$ - Mach-Zehnder interferometer.

When the driving voltage of the piezoelectric actuator was set to a $40 \mathrm{kHz}$ sinusoidal waveform with an amplitude of 4 Vpp, the calibrated strain on the fiber was $4.0 \mathrm{n} \varepsilon$ in amplitude. The received signal from coherent detection was collected uninterruptedly over a time length of $2 \mathrm{~ms}$. A portion of the received signal waveform (middle, red) was illustrated in Fig. 4, together with the synchronized light trigger pulse (top, blue) and acoustic drive signal (bottom, orange).

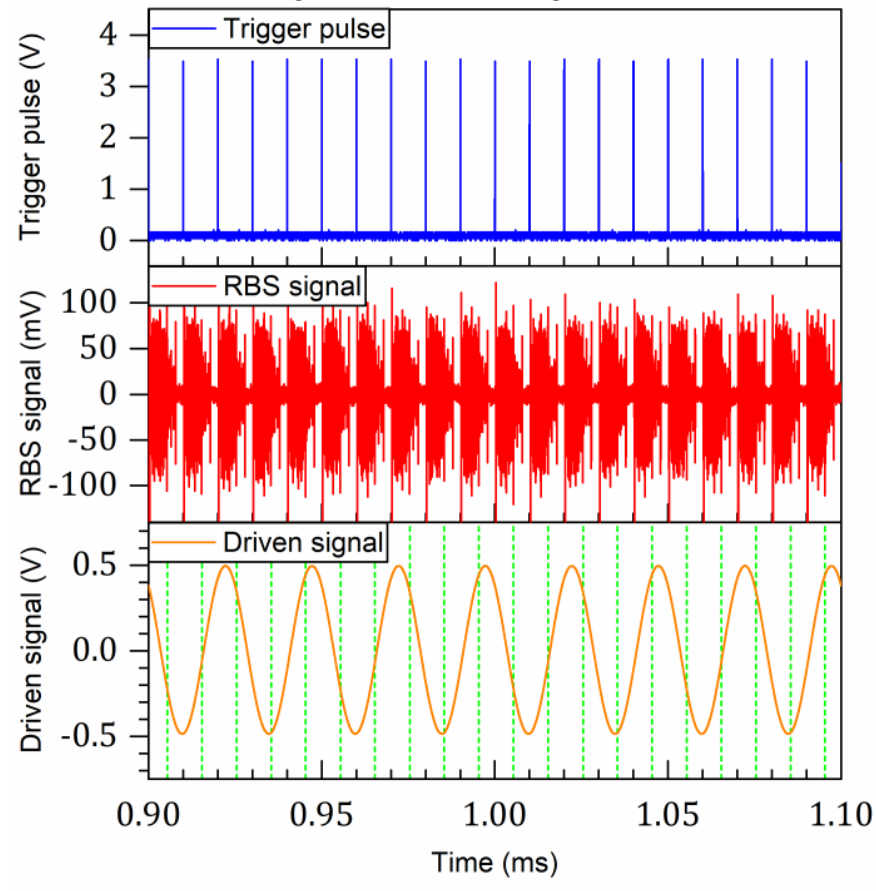

Fig. 4. A portion of 200 consecutive RBS received signal (middle, red), with the synchronized light trigger pulse (top, blue) and acoustic drive signal (bottom, orange). The trigger pulse period was $10 \mu \mathrm{s}$, and the acoustic frequency of the drive signal was $40 \mathrm{kHz}$.

First we adopted moving averaging and moving differential methods proposed in [15] to process the received $\Phi$-OTDR signal for the applied $4.0 \mathrm{n} \varepsilon$ single frequency dynamic strain. 
The RBS signal waveform was split and aligned with the light trigger pulse to form 200 raw traces, and the moving averaging number was 100. Then each of the averaged traces was subtracted by the reference zeroth averaged trace to obtain the detailed variations. Fig. 5(a) showed the experiment result. Due to the randomly distributed Rayleigh variate $A$ at each fiber location and the laser phase noise as discussed above, the RBS signal traces displayed intense fluctuations along the whole fiber length. As a result, the $4.0 \mathrm{n} \varepsilon$ dynamic strain perturbation applied to the fiber at the length range between $500 \mathrm{~m}$ and 600 $\mathrm{m}$ can hardly be identified.

We then used the proposed statistical estimator $\bar{R}$ in (18) to locate the acoustic perturbation area. The calculation of the statistical estimator involved four steps: 1) the whole received RBS waveform was split and aligned with the light trigger pulse in time to generate the sequenced $\Phi$-OTDR signal traces $\left.s_{i}(t), i=1,2, \cdots, 200.2\right)$ By applying Hilbert transform to each of the signal traces $s_{i}(t)$ to derive its conjugate function, the increment signal envelop $\left|\Delta s_{i}(t)\right|$ in (8) could be calculated, which exhibited the slowest rate of fluctuations in a meansquared sense. 3) We rearranged the increment signal envelop $\left|\Delta s_{i}(t)\right|=p(i \Delta t)$ (here, $\Delta t=10 \mu \mathrm{s}$ is the equivalent sampling interval of the acoustic perturbation signal, equaling the light pulse period), and applied the signal envelop calculation onto the acoustic perturbed signal $p(i \Delta t)$ in (15). Then we obtained the instantaneous amplitudes $R_{i}$ as a random variable following Rician density in (17). 4) The mean value of $R_{i}$ was calculated to obtain the statistical estimator $\bar{R}$ in (18).

The single-tone acoustic perturbation can be located via the $\bar{R}$ estimator by synthesizing all the 200 consecutive $\Phi$-OTDR signal traces, as shown in Fig. 5(b). Apart from the oscillations at the fiber entrance and reflecting end, there was a visible local peak at the fiber positions between $534.8 \mathrm{~m}$ and $540 \mathrm{~m}$, corresponding to the preset acoustic perturbation area. Note that there was a position shift of $25.8 \mathrm{~m}$ due to the initial light trigger time delay. By subtracting this shift, we located the perturbation area at the fiber positions between $509 \mathrm{~m}$ and $514.2 \mathrm{~m}$, which was wider than the preset location (from $512 \mathrm{~m}$ to $516 \mathrm{~m}$ ) and shifted forward by approximately $3 \mathrm{~m}$. The SNR of this local peak was measured as $5.1 \mathrm{~dB}$, for the $40 \mathrm{kHz}$ sinusoidal strain with amplitude of $4 \mathrm{n} \varepsilon$. The associated phase shift was calculated to be $0.018 \pi$, which was close to the minimum phase sensitivity determined by the laser phase noise of the $\Phi$-OTDR system.

Once the perturbed fiber section was located, the signal characteristics could be extracted by reconstructing the pertur-bation signal waveform in the time domain. As illustrated in Fig. 4, a set of equally spaced green dashed lines with a $10 \mu$ s spacing (must be equal to the repetition period of the launching pulse, corresponding to the same fiber position in the $\Phi$-OTDR traces), intersected with the acoustic drive signal at some specific points. Similarly, the $\Phi-O T D R$ traces played a role as a sequence of sampling points in the acoustic perturbation signal. For instance, at the peak location of $538.8 \mathrm{~m}$ in the inset of Fig. 5(b), we aligned all the $\Phi$-OTDR signal envelop traces $\left|\Delta s_{i}(t)\right|, i=1,2, \cdots, 200$. at the timestamp associated with the $538.8 \mathrm{~m}$ peak location and extracted the

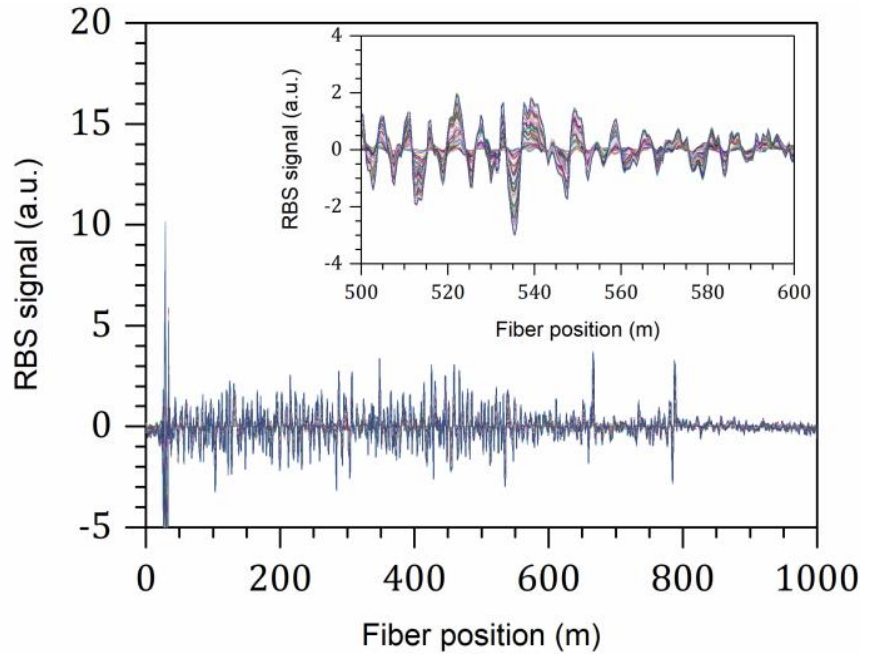

(a) The traditional superimposed plotting of OTDR traces by utilizing moving average and moving differential method. The moving averaging number is 100 . The inset depicts the local variation of the differential OTDR traces around the

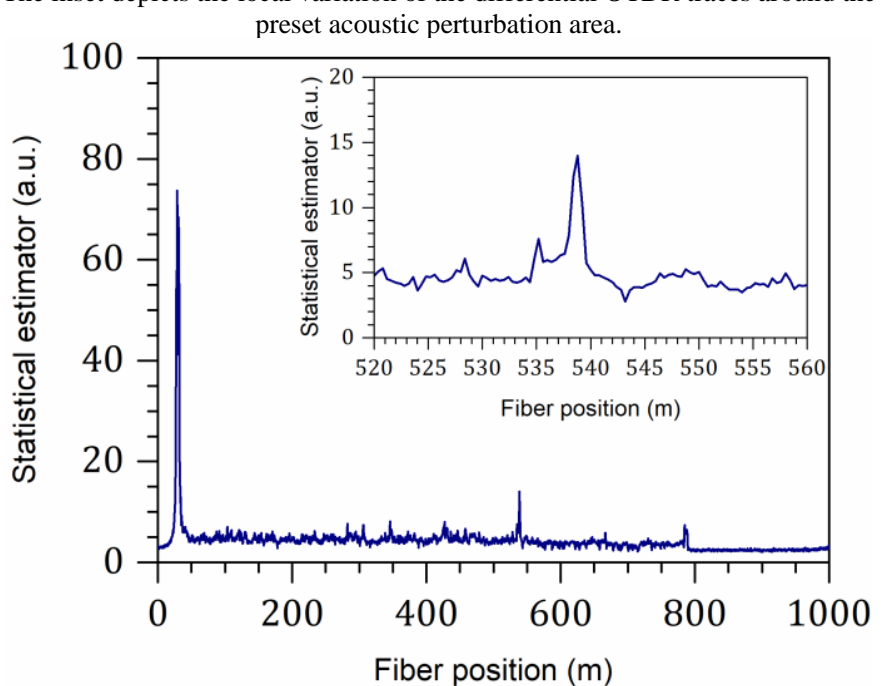

(b) Statistical estimator $\bar{R}$ curve vs fiber position by synthesizing $200 \Phi-O T D R$ traces. The inset describes the local peak around the fiber range between $520 \mathrm{~m}$ and $560 \mathrm{~m}$.

Fig. 5. Two approaches to process the received $\Phi$-OTDR signal. The fiber length was $764 \mathrm{~m}$. A $40 \mathrm{kHz}$ sinusoidal acoustic perturbation with a calibrated strain amplitude of $4.0 \mathrm{n} \varepsilon$ was set in the fiber region between $512 \mathrm{~m}$ and $516 \mathrm{~m}$. (Note that there was a position shift of $25.8 \mathrm{~m}$ due to the initial trigger time delay.)

instantaneous amplitude of the trace signal. Then, a rearranged waveform $p(i \Delta t), i=1,2, \cdots, 200$. was constructed in the time domain according to Nyquist sampling theorem, of which the sampling interval was $\Delta t=10 \mu \mathrm{s}$.

Fig. 6(a) illustrated the reconstructed acoustic waveform in the time domain, which exhibited many irregular fluctuations in the signal amplitude. Due to the poor SNR of the acoustic waveform, the signal features were hard to be recognized. By virtue of the Gabor spectrogram, the reconstructed acoustic signal $p(t)$ was transformed in the time-frequency domain. The calculated Gabor spectrogram was shown in Fig. 6(b), a persistent spectral content in the vicinity of $40 \mathrm{kHz}$ continued over time, which revealed the single-tone signature.

\section{B. Tone-burst signal test with $\Phi-O T D R$}

Let us draw our attention to the scenario of weak transient signal detection. For a partial discharge induced acoustic wave 


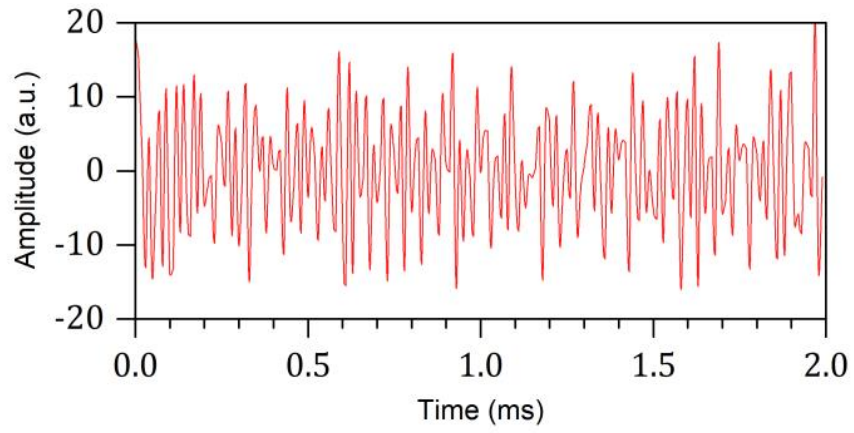

(a) The acoustic waveform in the time domain

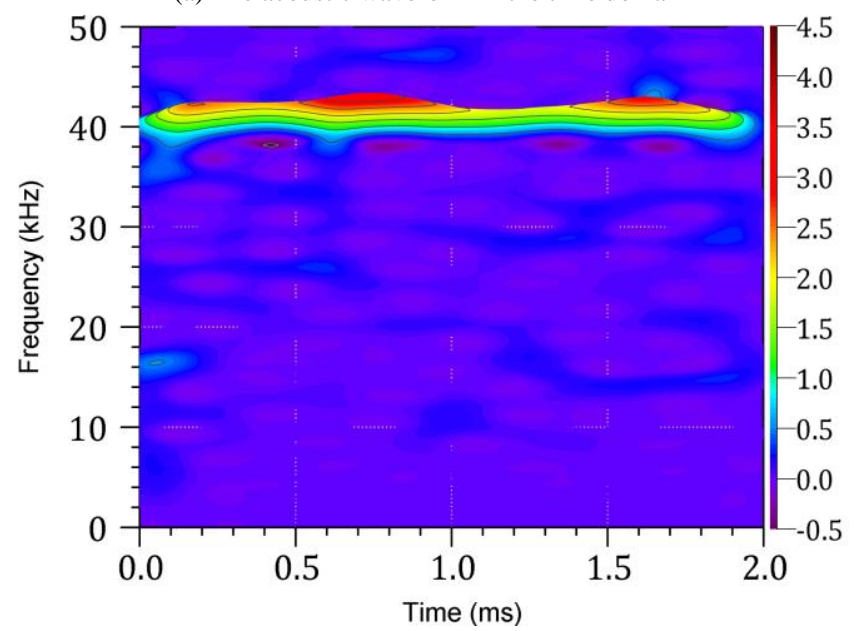

(b) Gabor spectrogram of the acoustic signal in the time-frequency domain Fig. 6. The reconstructed $40 \mathrm{kHz}$ single-tone acoustic signal at the $\Phi$-OTDR peak location of $538.8 \mathrm{~m}$ as shown in the inset of Fig. 5(b).

perturbation, it generally possesses burst wave behavior and usually occurs in a statistical manner with both the amplitude and the time of occurrence being stochastic. In this circumstance, both the location and the transient signal features of the acoustic-burst signal are to be detected with $\Phi-O T D R$.

In the experiment, we simulated the acoustic-burst signal by driving the piezoelectric actuator with a tone-burst electric signal. Fig. 7(a) depicted a case of four periods of a tone-burst signal with a duration of approximately $180 \mu \mathrm{s}$, a period of roughly $0.5 \mathrm{~ms}$, and a center frequency in the vicinity of $20 \mathrm{kHz}$. The amplitude of the burst signal was set to be $4 \mathrm{Vpp}$, while the $\mathrm{M}-\mathrm{Z}$ interferometer calibrated peak strain was $4.4 \mathrm{n} \varepsilon$. The statistical estimator $\bar{R}$ was measured in a similar way as described in the single-tone perturbation experiment. The estimator curve in the perturbation area of the fiber was shown in Fig. 7(b), the local peak was detected at the expected fiber positions.

The performance of the Gabor spectrogram in conjunction with $\Phi-O T D R$ for acoustic burst wave detection was illustrated as a new form of distributed mapping pattern shown in Fig. 7(c). The $\Phi-O T D R$ perturbation signal $p(t)$ was transformed according to (19) to obtain the Gabor coefficient $C_{m n}$ at each of the elementary points $(m T, n \Omega)$. By plotting the timefrequency estimator in (22) vs. time along each of the fiber positions, we identified the four periods of the acoustic-burst signal which behaved like isolated islands locating in the fiber region between $512 \mathrm{~m}$ and $518 \mathrm{~m}$ (Note that the $25.8 \mathrm{~m}$ position shift was already subtracted). The distributed mapping pattern was also beneficial to denoise the transient waveform subjected to Rayleigh fluctuations.

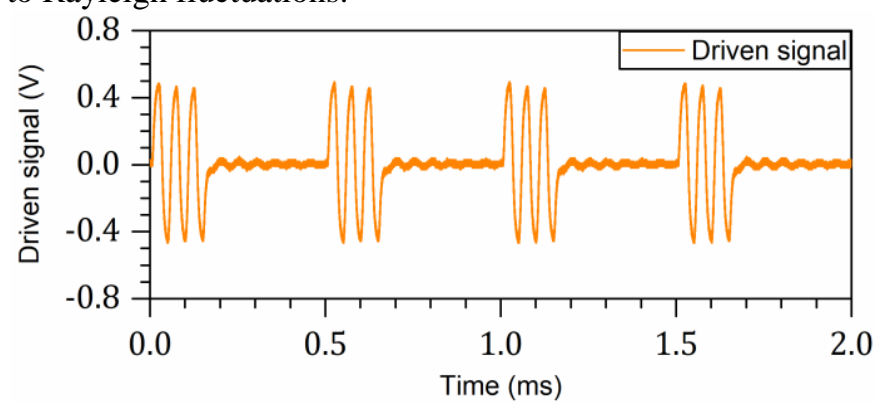

(a) The electrically driven tone-burst signal composing of periodic pulses, with

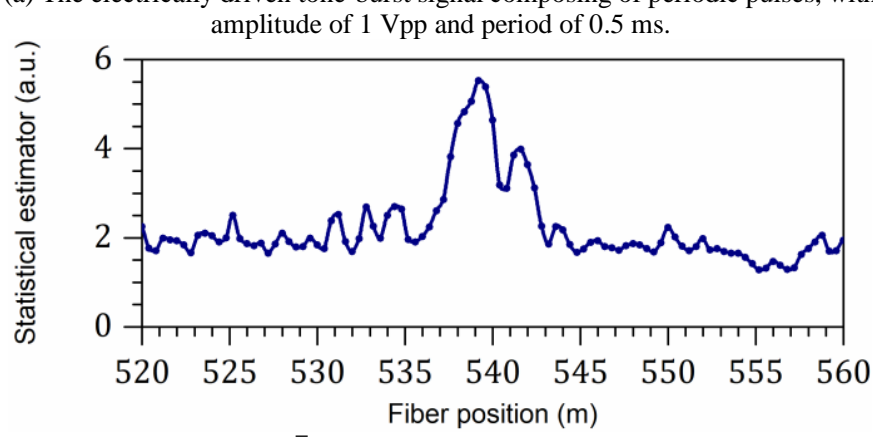

(b) Statistical estimator $\bar{R}$ curve vs fiber position (in part) for detecting and locating the acoustic burst wave.

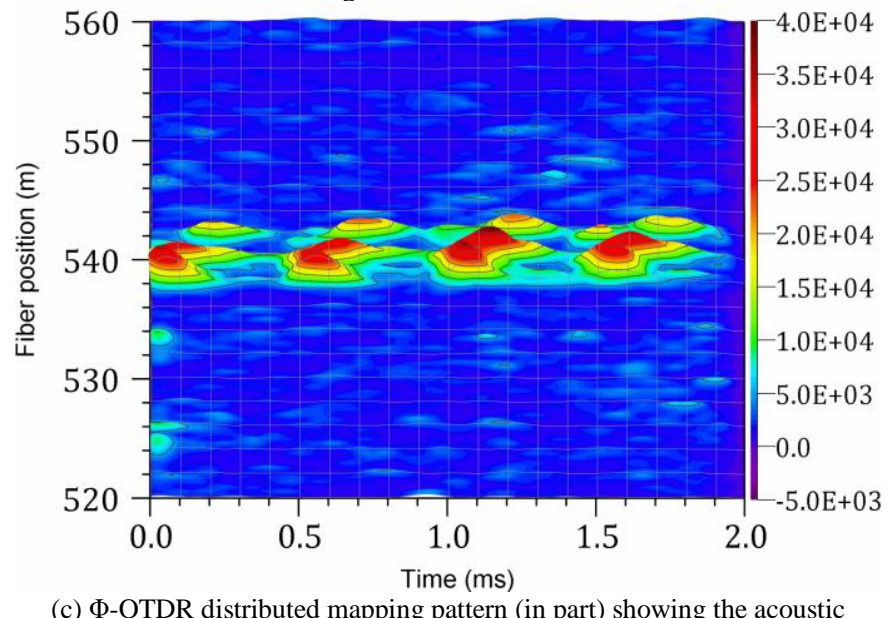

(c) $\Phi-O T D R$ distributed mapping pattern (in part) showing the acoustic tone-burst signal perturbation in the fiber region between $512 \mathrm{~m}$ and $518 \mathrm{~m}$.

(Note that the $25.8 \mathrm{~m}$ initial position shift should be subtracted).

Fig. 7. An example of acoustic burst wave detection using the cascaded statistical estimator and time-frequency estimator in $\Phi$-OTDR.

\section{DISCUSSION}

The $\Phi-O T D R$ measurement system needs a more sophisticated design to achieve better performance for the detection and location of ne-scale strain perturbations along the whole length of the fiber.

In coherent detection, the LO light field in the gain factors from (3) is effective when the LO power is increased to a threshold at which the LO shot noise dominates over all other noise terms for the detector. Fig. 8(a) showed the detectable SNR of the statistical estimator in (18) as a function of the LO power. There was a distinct turning area in which the SNR of the estimator rapidly increases up to $12 \mathrm{~dB}$ from a lower level $(6 \mathrm{~dB})$. This implied that the LO power larger than approximately $0.1 \mathrm{~mW}$ was sufficient to achieve shot-noise- 
limited detection, further increasing the LO power was not beneficial to the detection of SNR.

The effect of the pulse width was also considered. The test result shown in Fig. 8(b) illustrated that the SNR of the statistical estimator exhibited just a slight increasing tendency, which was $3 \mathrm{~dB}$ with the pulse width increasing from $30 \mathrm{~ns}$ to $100 \mathrm{~ns}$, while the input light power increased as well. However, we must consider the tradeoff between the SNR improvement and the deterioration of the detection spatial resolution. This is because the SNR improvement achieved through broadening the pulse results a relatively lower spatial resolution for the $\Phi-O T D R$ distributed system. In addition, the broadened pulse width gives rise to a much larger variance of the laser phase noise as discussed above.

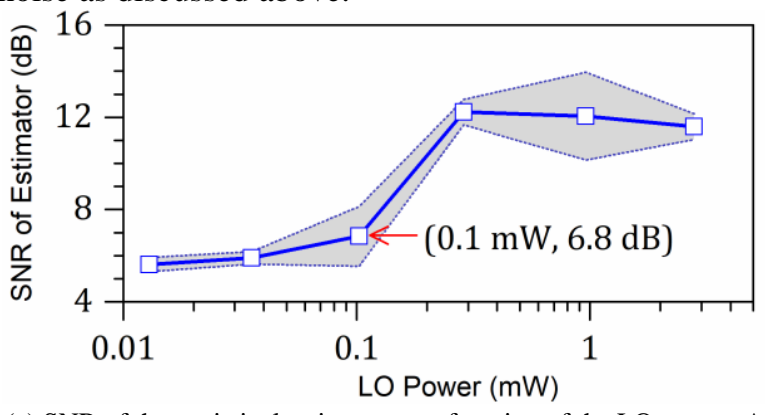

(a) SNR of the statistical estimator as a function of the LO power. A series of

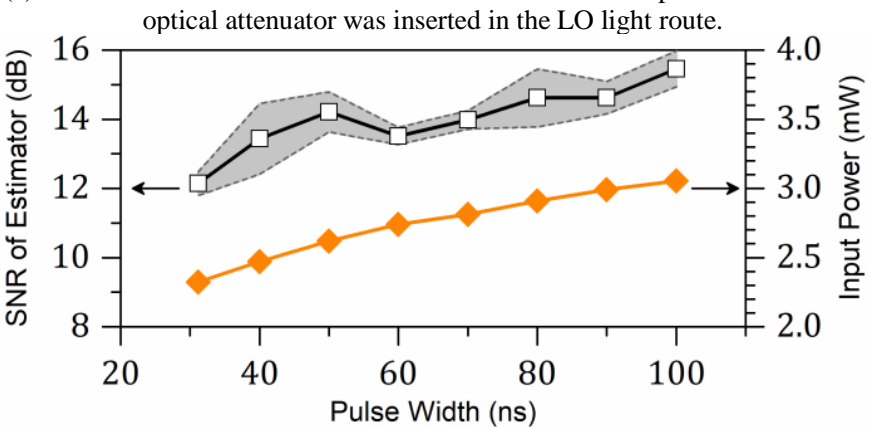

(b) SNR of the statistical estimator as a function of the pulse width of the probe light. The relationship between the input power of the pulsed light and pulse width was also presented.

Fig. 8. Key parameters that affect the performance of the $\Phi$-OTDR measure-ment system. The test data were obtained by utilizing a $4.0 \mathrm{n} \varepsilon, 40 \mathrm{kHz}$ sinusoidal dynamic strain perturbation. The shaded region within the dashed line indicated the multiple measurement variations.

Overall, we believe that the proposed improvement in $\Phi$-OTDR could pave the way for the detection of partial discharge and associated faults for high-voltage equipment, which has drawn much research interest in the field of fiber-optic sensors [27-31]. A summary of the frequency band and magnitude level of some common discharging phenomena is shown in Table I. It can be seen that the majority of discharges actually have frequency components ranging from audible frequencies to $100 \mathrm{kHz}$, which means that our proposed system can partially meet the requirements. It is mentioned that the $\Phi$-OTDR technique still does not possess the required sensitivity for the detection of partial discharge induced acoustic emission waves, which may be 1 2 orders of magnitude lower than the minimal detectable strains examined in the preceding experiments. In [28], the authors have reported the acoustic detection of partial discharges in a high-voltage power cable termination by utilizing a commercially available
C-OTDR instrument. Despite a detection limit of $1 \mathrm{nC}$ achieved with a dedicated ultrasonic fiber-optic transducer, the magnitude detection sensitivity in $\mathrm{nC}$ scale cannot meet the requirement of in situ PD monitoring of power cables. Future studies can further improve the performance of $\Phi$-OTDR from all aspects, including fundamental fiber sensor materials and advanced signal processing algorithms, to make the system competitive in the discharging detection applications.

$$
\text { TABLE I }
$$

A SUMMARY OF THE FREQUENCY BANDS AND MAGNITUdE LEVEL OF THE COMMENT DISCHARGING PHENOMENA

\begin{tabular}{llc}
\hline \hline \multicolumn{1}{c}{ Types of Discharging } & Frequency Bands & $\begin{array}{c}\text { Magnitude } \\
\text { Levels }\end{array}$ \\
\hline Thunder from lightning & $10 \mathrm{~Hz} \sim 2 \mathrm{kHz}$ & $10^{6} \mathrm{~Pa}$ \\
Corona & $50 \mathrm{~Hz} \sim 300 \mathrm{kHz}$ & $10^{-2} \mathrm{~Pa}$ \\
Discharge on transmission lines & $10 \mathrm{kHz} \sim 100 \mathrm{kHz}$ & $10^{-3} \mathrm{~Pa}$ \\
Discharge in solid insulation & $20 \mathrm{kHz} \sim 2 \mathrm{MHz}$ & $*$ lack of data \\
Discharge in liquid insulation & $20 \mathrm{kHz} \sim 300 \mathrm{kHz}$ & $10^{0} \mathrm{~Pa}$ \\
Discharge in gaseous insulation & $10 \mathrm{kHz} \sim 80 \mathrm{kHz}$ & $10^{-3} \mathrm{~Pa}$ \\
Discharge at low pressure and in & $20 \mathrm{kHz} \sim 80 \mathrm{kHz}$ & $10^{-3} \mathrm{~Pa}$ \\
vacuum insulation & \\
\hline \hline
\end{tabular}

\section{V.CONCLUSION}

This paper focused on the feasibility of $\Phi$-OTDR sensing technology for the distributed detection of acoustic wave induced $\mathrm{n} \varepsilon$ or sub-n $\varepsilon$ transient weak strains upon a fiber. Coherent detection was employed in the $\Phi$-OTDR configuration to improve the sensitivity and obtain a much better SNR. A cascaded signal processing framework was proposed, consisting of a statistical estimator that is used to capture and locate the target signal, and a time-frequency estimator that extracts the temporal and frequency features of the signal for determination. Experimental tests were implemented to verify the improved $\Phi$-OTDR system. It was demonstrated that the $\Phi$-OTDR system that adopted this signal processing framework was capable of detecting and locating dynamic strain with a magnitude down to $4 \mathrm{n} \varepsilon$ and an acoustic frequency up to $40 \mathrm{kHz}$. A tone-burst acoustic signal, analogous to the acoustic waves induced by partial discharge phenomena in electrical insulation, was also tested to prove the promising potential for transient weak signal detections. Future work must be carried out to design a more sensitive and reliable $\Phi-O T D R$ distributed fiber sensing system. Nevertheless, the proposed statistic signal-processing based $\Phi$-OTDR technology holds the promise for the remote and distributed detection of partial discharge in long-distance power cables.

\section{ACKNOWLEDGMENT}

The authors extend great thanks to Fuji Ceramics for the custom design of the piezoelectric actuator, Qingwen Liu for his support on the dynamic strain test of fibers, and Jingfei Li and Zhen'guo Zhang for their helpful work.

\section{REFERENCES}

[1] F. Teng, D. Yi, X. M. Hong, and X. J. Li, "Distributed fiber optics disturbance sensor using a dual-Sagnac interferometer," Optics Letters, vol. 44, no. 20, pp. 5101-5103, Oct 152019. 
[2] Y. Li, X. Z. Wang, and X. Y. Bao, "Sensitive acoustic vibration sensor using single-mode fiber tapers," Applied Optics, vol. 50, no. 13, pp. 1873-1878, May 12011.

[3] J. S. Jia, Y. Jiang, L. C. Zhang, H. C. Gao, and L. Jiang, "Fiber Optic Dual-Ring Michelson Interferometer-Based Detection Scheme for the Measurement of Dynamic Signals," Journal of Lightwave Technology, vol. 37, no. 15, pp. 3750-3755, Aug 12019.

[4] M. J. Murray, A. Davis, and B. Redding, "Fiber-Wrapped Mandrel Microphone for Low-Noise Acoustic Measurements," Journal of Lightwave Technology, vol. 36, no. 16, pp. 3205-3210, Aug 15 2018

[5] W. J. Ni et al., "Ultrathin graphene diaphragm-based extrinsic Fabry-Perot interferometer for ultra-wideband fiber optic acoustic sensing," Optics Express, vol. 26, no. 16, pp. 20758-20767, Aug 6 2018.

[6] Q. Wu, Y. Okabe, and J. H. Wo, "Fiber Sensor Based on Interferometer and Bragg Grating for Multiparameter Detection," IEEE Photonics Technology Letters, vol. 27, no. 12, pp. 1345-1348, Jun 152015.

[7] J. Y. Wang et al., "Diaphragm-based optical fiber sensor array for multipoint acoustic detection," Optics Express, vol. 26, no. 19, pp. 25293-25304, Sep 172018.

[8] G. H. Kim, S. M. Park, C. H. Park, H. Jang, C. S. Kim, and H. D. Lee, "Real-time quasi-distributed fiber optic sensor based on resonance frequency mapping," Scientific Reports, vol. 9, Mar 8 2019.

[9] Z. Y. Li et al., "Simultaneous distributed static and dynamic sensing based on ultra-short fiber Bragg gratings," Optics Express, vol. 26, no. 13, pp. 17437-17446, Jun 252018.

[10] Y. P. Liu et al., "A Feasibility Study of Transformer Winding Temperature and Strain Detection Based on Distributed Optical Fibre Sensors," Sensors, vol. 18, no. 11, pp.3932, Nov 2018.

[11] Y. Peled, A. Motil, and M. Tur, "Fast Brillouin optical time domain analysis for dynamic sensing," Optics Express, vol. 20, no. 8, pp. 8584-8591, Apr 92012.

[12] B. Wang, X. Y. Fan, Y. X. Fu, and Z. Y. He, "Dynamic strain measurement with kHz-level repetition rate and centimeter-level spatial resolution based on Brillouin optical correlation domain analysis," Optics Express, vol. 26, no. 6, pp. 6916-6928, Mar 19 2018.

[13] Z. Y. Zhang and X. Y. Bao, "Distributed optical fiber vibration sensor based on spectrum analysis of Polarization-OTDR system," Optics Express, vol. 16, no. 14, pp. 10240-10247, Jul 72008.

[14] Z. Y. Zhang and X. Y. Bao, "Continuous and damped vibration detection based on fiber diversity detection sensor by Rayleigh backscattering," Journal of Lightwave Technology, vol. 26, no. 5-8, pp. 832-838, Mar-Apr 2008.

[15] Y. L. Lu, T. Zhu, L. A. Chen, and X. Y. Bao, "Distributed Vibration Sensor Based on Coherent Detection of Phase-OTDR," Journal of Lightwave Technology, vol. 28, no. 22, pp. 3243-3249, Nov 15 2010.

[16] J. Pastor-Graells, H. F. Martins, A. Garcia-Ruiz, S. Martin-Lopez, and M. Gonzalez-Herraez, "Single-shot distributed temperature and strain tracking using direct detection phase-sensitive OTDR with chirped pulses," Optics Express, vol. 24, no. 12, pp. 13121-13133, Jun 132016.

[17] A. Masoudi and T. P. Newson, "High spatial resolution distributed optical fiber dynamic strain sensor with enhanced frequency and strain resolution," Optics Letters, vol. 42, no. 2, pp. 290-293, Jan 15 2017.

[18] T. Zhu, X. H. Xiao, Q. He, and D. M. Diao, "Enhancement of SNR and Spatial Resolution in phi-OTDR System by Using Two-Dimensional Edge Detection Method," Journal of Lightwave Technology, vol. 31, no. 17, pp. 2851-2856, Sep 12013.

[19] S. Zhou, F. Hao, and Z. M. Zeng, "Phase demodulation method in phase-sensitive OTDR without coherent detection," Optics Express, vol. 25, no. 5, pp. 4831-4844, Mar 62017.

[20] D. Chen, Q. W. Liu, X. Y. Fan, and Z. Y. He, "Distributed Fiber-Optic Acoustic Sensor With Enhanced Response Bandwidth and High Signal-to-Noise Ratio," Journal of Lightwave Technology, vol. 35, no. 10, pp. 2037-2043, May 152017.

[21] X. Y. Bao, D. P. Zhou, C. Baker, and L. Chen, "Recent Development in the Distributed Fiber Optic Acoustic and Ultrasonic Detection," Journal of Lightwave Technology, vol. 35, no. 16, pp. 3256-3267, Aug 152017.
[22] R. T. Harrold, "Acoustical Technology Applications in Electrical Insulation and Dielectrics," IEEE Transactions on Electrical Insulation, vol. 20, no. 1, pp. 3-19, 1985.

[23] Z. Q. Pan, K. Z. Liang, Q. Ye, H. W. Cai, R. H. Qu, and Z. J. Fang, "Phase-sensitive OTDR system based on digital coherent detection," In Optical Sensors and Biophotonics III, Proc. SPIE, vol. 8311, Shanghai, China, 13-16 Nov, 2011, pp. 83110S.

[24] J. W. Goodman, Speckle phenomena in optics : theory and applications, 2nd ed. vol. PM312. Bellingham, USA: SPIE, 2020.

[25] K.-P. Ho, Phase-modulated optical communication systems. New York, USA: Springer, 2005.

[26] B. Friedlander and A. Zeira, "Oversampled Gabor Representation for Transient Signals," IEEE Transactions on Signal Processing, vol. 43, no. 9, pp. 2088-2094, Sep 1995.

[27] P. Rohwetter, R. Eisermann, and K. Krebber, "Random Quadrature Demodulation for Direct Detection Single-Pulse Rayleigh C-OTDR," Journal of Lightwave Technology, vol. 34, no. 19, pp. 4437-4444, Oct 12016.

[28] P. Rohwetter, R. Eisermann, and K. Krebber, "Distributed acoustic sensing: Towards partial discharge monitoring," in OFS-24, 24th International Conference on Optical Fibre Sensors, Proc. SPIE, vol. 9634, Curitiba, Brazil, 28 Sep-2 Oct, 2015, pp. 9634 1C.

[29] G. M. Ma, C. Shi, W. Q. Qin, Y. B. Li, H. Y. Zhou, and C. R. Li, "A Non-Intrusive Electrical Discharge Localization Method for Gas Insulated Line Based on Phase-Sensitive OTDR and Michelson Interferometer," IEEE Transactions on Power Delivery, vol. 34, no. 4, pp. 1324-1331, Aug 2019.

[30] W. Pan, K. Zhao, C. Xie, X. Li, J. Chen, and L. Hu, "Distributed Online Monitoring Method and Application of Cable Partial Discharge Based on $\varphi$-OTDR," IEEE Access, vol. 7, pp. 144444-144450, 2019.

[31] L. Kirkcaldy, J. Pilgrim, R. Rogers, and G. Lees, "Distributed Acoustic Sensing of Partial Discharge: Initial Findings," in Jicable'19, 10th International Conference on Insulated Power Cables, Paris-Versailles, France, 23-27 Jun, 2019, pp. F2-11.

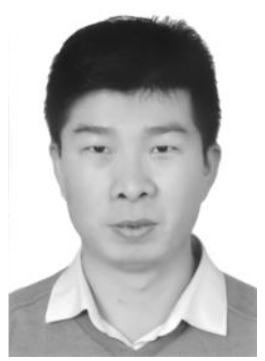

Hao Chen was born in Xi'an, China in 1981. He received a B.S. degree in applied physics and a M.S. degree in electronic physics from Xi'an Jiaotong University, Xi'an, China, in 2004 and 2008, respectively. $\mathrm{He}$ is currently pursuing a $\mathrm{Ph} . \mathrm{D}$. degree in electrical engineering in Xi'an Jiaotong University, China. His research interests are power equipment condition monitoring and fiber optic sensors.

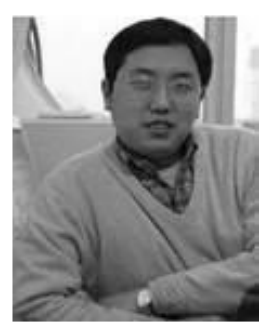

Yang Xu was born in Xi'an, China in 1969. He received B.S., M.S. and Ph.D. degrees in electrical engineering from $\mathrm{Xi}^{\prime}$ an Jiaotong University (XJTU), Xi'an, China. $\mathrm{He}$ is now a professor of XJTU. His research interest lies in partial discharge detection and new insulation material for power equipment. He is an AG, WG4 and WG6 member of the IEC TC112 "Evaluation and Qualification of Electrical Insulating Materials and Systems". He is also a member of the CIGRE WG B1.28 "On-site Partial Discharge Assessment”. 


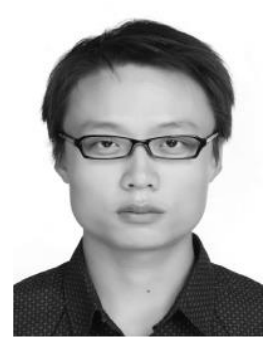

ester insulating oil.

Sen Qian received B.S., M.S., and Ph.D. degrees in electrical engineering from Xi'an Jiaotong University, Xi'an, China. $\mathrm{He}$ is currently a researcher in Guangzhou Institute of Advanced Technology, Chinese Academy of Science, Guangzhou, China. His research interests are power equipment monitoring, fiber optic sensor, fiber optics, dielectric liquid and natural

Hai Yuan received his Ph.D. degree in Electrical and Electronic Engineering from Nanyang Technological University in 2006. He is currently a professor in Shenzhen Institute of Advanced Technology, Chinese Academy of Science and also the director of Guangzhou Institute of Advanced Technology, Chinese Academy of Science. He is currently the vice team leader of Pattern Recognition and Intelligent System Research Group, and his main research interests include fiber optic communication and sensor, wireless networks. Prof. Yuan is an IEEE senior member and also serves as the vice president of alumni association of Nanyang Technological University in Guangdong Province.

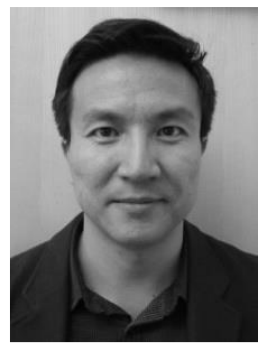

Lei Su received B.S. and M.S. degrees from Xi'an Jiaotong University, Xi'an, China, in 1999 and 2002, respectively, and a Ph.D. degree from Nanyang Technological University, Singapore, in 2006. This was followed by postdoctoral trainings at the University of Cambridge and Imperial College London. $\mathrm{He}$ is currently a Senior Lecturer in the School of Engineering and Materials Science at Queen Mary University of London. His research interests include optical imaging, sensing and instrumentation. 